\title{
SOBRE ARBITRAJE ADMINISTRATIVO: EL CASO DE LAS CONTRATACIONES DE MENOS DE 8 UIT
}

\author{
Oscar HerRera GiURFA †* \\ Universidad de Lima, Lima, Perú \\ Recibido: 9/8/2020 Aceptado: 16/8/2021 \\ doi: https://doi.org/10.26439/iusetpraxis2021.n053.4950
}

RESUMEN. La forma prevista por el ordenamiento jurídico peruano para contratar con las entidades y empresas del Estado se encuentra en la Ley de Contrataciones; pero en esta norma existen supuestos que permiten su inaplicación, los cuales se dividen en dos grupos: (i) aquellos en los que no se requiere dar cuenta al OSCE de las contrataciones realizadas, como los contratos bancarios o la contratación de notarios, y (ii) las contrataciones que requieren la supervisión del OSCE, como las contrataciones de hasta 8 UIT. Al revisar la Ley de Contrataciones, su reglamento, las directivas y las opiniones del OSCE, podemos advertir que el marco legal de las contrataciones de hasta 8 UIT no se encuentra claramente delimitado, más allá del registro de las mismas en el SEACE, tal como dispone el literal a) del artículo 11.2 de la Directiva 008-2017-0SCE/ CD, "Disposiciones aplicables al registro de información en el Sistema Electrónico de Contrataciones del Estado - SEACE". No obstante, y tal como podemos advertir, regular únicamente la forma de registrar la información de estas contrataciones, dejando de lado aspectos sustanciales como cuál es la normativa aplicable para estas contrataciones y cómo se resolverán las controversias de las mismas, resulta insuficiente. En tal sentido y considerando la importancia de los temas planteados, el presente artículo aborda la posibilidad de incluir un arbitraje administrativo especial como medio de solución efectivo de controversias en las contrataciones de hasta 8 UIT.

\section{PALABRAS CLAVE: administrativo / arbitraje / contrataciones / controversias / solución / contrato}

\footnotetext{
* Abogado por la Pontificia Universidad Católica del Perú. Máster en Derecho Administrativo Económico por el Instituto Ortega y Gasset, adscrito a la Universidad Complutense de Madrid, España. Profesor de la Universidad de Lima, Universidad ESAN y Universidad San Ignacio de Loyola. Árbitro del Listado de Neutrales del OSCE, del Centro de Arbitraje de la Cámara de Comercio de Lima y del Centro de Arbitraje de la Cámara de Comercio de San Martín. Lamentablemente, Oscar Herrera Giurfa falleció el 6 de febrero del 2021.
} 


\section{ABOUT ADMINISTRATIVE ARBITRATION: THE CASE OF CONTRACTING UP TO EIGHT ITUS}

ABSTRACT. The way foreseen by the Peruvian legal system to contract with State entities and companies is by using the Procurement Law; however, in the said regulation, some assumptions allow its non-application. These exceptional cases are divided into two groups: (i) those in which it is not required to report to the OSCE the contracting carried out, as is the case of bank contracts or the hiring of notaries, and (ii) contracts that require supervision of OSCE, as is the case of the recruitment of up to eight ITU. When reviewing the Procurement Law, its Regulations, the OSCE Directives, and Opinions, we can see that the legal framework for contracting up to 8 ITUs is not delimited, beyond their registration in SEACE, such as provided in literal a) of article 11.2 of Directive 008-2017-OSCE / CD "Provisions applicable to the registration of information in the Electronic System of State Procurement - SEACE". However, and as we can warn, to regulate only the way of recording the information of these contracts, leaving aside substantial aspects such as what is the applicable regulations for these contracts is not enough. And how will their controversies be resolved? It is insufficient. In this sense, and considering the importance of the issues raised in this article, it will address the possibility of including administrative arbitration, as an alternative for effective dispute resolution, in the contracting of up to eight ITUs.

KEYWORDS: administrative / arbitration / contracting / controversy / solution / contract 


\section{INTRODUCCIÓN}

La Ley 26850, Ley de Contrataciones y Adquisiciones del Estado, publicada el 30 de julio de 1997, fue el primer texto único ordenado de la Ley de Contrataciones en el Perú. Fue el primer texto normativo que ordenó los sistemas de contrataciones, ya que agrupó a los tres sistemas con los que se realizaban las contrataciones en esa época: el Reglamento Único de Licitaciones y Contratos de Obras Públicas (RULCOP), el Reglamento Único de Adquisiciones para el Suministro de Bienes y la Prestación de Servicios No Personales (RUA) y el Reglamento General de Asesorías y Consultorías (REGAC).

La acotada normativa contenía los siguientes procedimientos de selección: licitación pública, concurso público, adjudicación directa y de menor cuantía, los cuales permitían poder elegir la mejor alternativa dentro de las propuestas presentadas por los postores interesados. Luego, se procedía a la suscripción del contrato correspondiente. El artículo 53 de la mencionada Ley 26850 (1997) estableció:

Las controversias que surjan sobre la ejecución o interpretación del contrato se resolverán obligatoriamente mediante los procedimientos de arbitraje o conciliación. Si la conciliación concluyera con un acuerdo parcial o sin acuerdo, las partes deberán someterse a arbitraje para que se pronuncie sobre las diferencias no resueltas o resuelva la controversia definitivamente. Como se puede apreciar, el arbitraje también resultaba de aplicación para los contratos (órdenes de compra u órdenes de servicio) derivados de una menor cuantía.

El actual texto único ordenado de la Ley de Contrataciones del Estado, aprobada mediante el Decreto Supremo 082-2019-EF, publicado el 13 de marzo del 2019, reconoce como procedimientos de selección: la licitación pública, el concurso público, la adjudicación simplificada, la selección de consultores individuales y la comparación de precios. Estos procedimientos sirven a las entidades públicas para poder elegir la mejor alternativa entre las propuestas presentadas por los postores interesados, luego de lo cual se procede a la suscripción del contrato correspondiente. Adicionalmente, el artículo 45 del acotado TUO indica:

Las controversias que surjan entre las partes sobre la ejecución, interpretación, resolución, inexistencia, ineficacia o invalidez del contrato se resuelven, mediante conciliación o arbitraje, según el acuerdo de las partes. En el reglamento se definen los supuestos para recurrir al arbitraje ad hoc. Las controversias sobre la nulidad del contrato solo pueden ser sometidas a arbitraje. Como se puede leer, el arbitraje resulta de aplicación para los contratos surgidos en aplicación de los procedimientos antes mencionados. (Decreto Supremo 082-2019-EF, 2019)

Debemos señalar que, después de más de veinte años de aplicación de la Ley de Contrataciones para satisfacer las necesidades de bienes, servicios y obras de las entidades públicas, se sigue utilizando un cuerpo legal especial para dichas contrataciones, tomando el modelo francés y recogiendo supuestos como el de interés público, 
recursos públicos y transparencia. No obstante, en la actualidad hay algunos supuestos de contratación que escapan a la aplicación de la ley especial, como es el caso de las contrataciones de hasta 8 UIT, que requieren una regulación especial. En algunos casos, esta se desarrolla a través de directivas, pero lamentablemente en la mayoría de las veces no tiene regulación alguna y, en tal sentido, tampoco se encuentra establecido mecanismo alguno de solución de controversias.

\section{EL CONTRATO ADMINISTRATIVO}

La Administración necesita de la colaboración de los particulares para poder cumplir muchas de las obligaciones que le son propias, en algunos casos porque le es imposible realizarlas por sí misma, y en otros, debido a la necesidad de eficiencia económica que solo se consigue con la contratación de particulares. La doctrina ha desarrollado una serie de posiciones sobre la naturaleza de los contratos que celebra la Administración y el régimen aplicable a estos.

En ese sentido, consideramos pertinente compartir algunas definiciones respecto al contrato administrativo. A decir de Diego Zegarra, "el contrato administrativo es todo acuerdo generador de obligaciones celebrado por un órgano estatal, en ejercicio de la función administrativa, susceptible de producir efectos con relación a terceros" (como se citó en Trelles, 2002, p. 238). Así, para diferenciar el contrato administrativo del privado, tenemos el criterio material, que distingue como elemento diferenciador del contrato administrativo el interés público en juego; y el criterio de las cláusulas exorbitantes, según el cual el hecho de que se incluya este tipo de cláusulas en un contrato con la Administración lo convierte en administrativo. Según Linares (2006):

La concepción clásica del contrato administrativo apareja la existencia de potestades que trascienden la letra misma del contrato, siendo por tanto implícitas y de aplicación incluso en el caso en que se haya pactado en contra de ellas. Las potestades son de modificación unilateral del contrato, de interpretación unilateral y ejecutoria, de dirección y control, sancionadoras y resolutorias; se puede incluir, además, la inaplicación de la excepción de incumplimiento. Se fundan en la tutela que debe ejercer el Estado respecto del interés público comprometido. (p. 286)

Por su parte, De la Puente (1991) define el contrato administrativo como "el acuerdo de voluntades, generador de obligaciones, celebrado entre un órgano del Estado, en ejercicio de las funciones administrativas que le competen, con otro órgano administrativo o con un particular" (pp. 361-362). Como criterios para caracterizar a un contrato como administrativo, opta por el de su objeto y por contener cláusulas exorbitantes del derecho privado. En similar sentido, Amado y Miranda (2000) sostienen:

Un contrato es administrativo por razón de su objeto o por contener cláusulas exorbitantes al derecho privado, y que el hecho [de] que un contrato sea administrativo 
conlleva que la relación jurídico-patrimonial surgida de este se rija por el derecho público. (p. 258)

Para concluir las definiciones del contrato administrativo, tenemos la de Salazar (2009), quien explica lo siguiente:

[...] la relación contractual vincula a dos (o más) sujetos, aunque en este caso se presentan dos posibilidades: (a) uno de ellos ejerce poder (específicamente función administrativa del poder) y el otro su libertad, o (b) uno de los sujetos ejerce poder y el otro sujeto también ejerce poder. En tal sentido, la relación subyacente a los contratos de la Administración Pública es la de poder con libertad o la de poder con poder; de lo cual se deriva que la construcción del régimen contractual público debe girar en torno a dichas posibilidades de vinculación, estableciendo los principios y normas que garanticen las facultades, deberes, derechos y obligaciones involucrados en tales relaciones. (p. 26)

Particularmente, entendemos que el contrato de la Administración es el género, en la medida en que esta puede contratar inclusive sin utilizar una norma propia, como en el caso de los contratos suscritos con el sistema financiero; y que el contrato administrativo es la especie, en cuyo caso una entidad siempre debe utilizar una norma especial, como es el caso de las contrataciones realizadas utilizando la Ley de Contrataciones del Estado. Adicionalmente, consideramos como elementos diferenciadores de los contratos administrativos a la formalidad que conlleva la suscripción del mismo, así como el haber desarrollado un procedimiento previo, la competencia del funcionario que lo suscribe y el hecho de que detrás de cada contrato administrativo siempre existe interés público. Finalmente, uno de los elementos diferenciadores de los contratos administrativos son las prerrogativas que tiene la Administración y que no tiene el contratista, como es el caso de poder elaborar las condiciones del contrato, la aplicación de las penalidades por mora y las otras penalidades, la capacidad de disponer sobre las prestaciones adicionales o las reducciones contractuales, entre otros, supuestos que ponen en evidencia que si bien estamos ante una relación contractual, las partes que la conforman no son iguales. No obstante, la Ley de Contrataciones ha dispuesto que las controversias que se susciten durante la ejecución contractual serán resueltas mediante arbitraje, y no mediante un mecanismo administrativo.

\section{LOS SUPUESTOS DE INAPLICACIÓN DE LA LEY DE CONTRATACIONES}

El artículo 3 de la Ley de Contrataciones establece los supuestos en los que esta debe ser empleada por las entidades para contratar, así como la relación de entidades que deben hacerlo. De esta manera, tenemos que la totalidad de entidades, organismos públicos, organismos constitucionalmente autónomos, empresas públicas, gobiernos regionales, provinciales, locales y otros que utilicen fondos públicos se encuentran obligados a utilizarla; no obstante, los artículos 4 y 5 de la precitada norma establecen 
aquellos supuestos que se encuentran excluidos del ámbito de aplicación de la misma, los cuales se dividen en dos grupos: aquellos sin supervisión del Organismo Supervisor de las Contrataciones del Estado (OSCE) (previstos en el artículo 4), como los contratos bancarios y financieros, las contrataciones de notarios públicos, los servicios brindados por conciliadores, árbitros o miembros de la junta de resolución de disputas; y aquellos sujetos a la supervisión del OSCE, en los que encontramos los servicios públicos, los convenios suscritos entre entidades, las contrataciones realizadas entre Estados y las contrataciones de hasta 8 UIT.

En los casos antes descritos, las contrataciones que se enmarquen dentro de los supuestos excluidos del ámbito de aplicación de la normativa de contrataciones del Estado previstos en la Ley de Contrataciones o en otra ley (como en el caso de las contrataciones realizadas en el marco de los Juegos Panamericanos), así como aquellas actuaciones que no reúnan las características de una contratación con el Estado, podrán realizarse sin observar las disposiciones de dicha normativa; no obstante, tal supuesto no enerva la obligación de observar los principios que rigen toda contratación pública, considerándolos independientemente de que no se utilice la Ley de Contrataciones, pues implican la erogación de fondos públicos.

De lo expuesto, debemos tener presente que aquellas contrataciones que se enmarquen dentro de los supuestos de inaplicación de la normativa de contrataciones del Estado o no reúnan las características de una contratación pública dentro de su ámbito podrán realizarse sin observar sus disposiciones.

\section{LAS CONTRATACIONES DE HASTA 8 UIT}

En la Ley 26850 existía la adjudicación de menor cuantía, que se utilizaba para contrataciones desde una unidad impositiva tributaria (UIT) hasta los 15000 soles, en el caso de servicios; hasta los 35000 soles para bienes; y hasta los 90000 soles para obras. En ese sentido, se puede advertir que el legislador dispuso la aplicación de la ley de contrataciones para procedimientos realizados a partir de 1 UIT, dejando libertad contractual respecto a aquellos supuestos que se realizaran por montos inferiores. No obstante, con el transcurrir de los años, la política respecto a las contrataciones sin aplicación de la normativa de contrataciones se fue flexibilizando, y en la actualidad, conforme lo dispone el artículo 5 de la Ley de Contrataciones, los supuestos que van hasta 8 UIT (34 400 soles, al momento de elaborar este artículo) están excluidos del ámbito de aplicación de la mencionada ley.

En junio del año 2008, mediante el Decreto Legislativo 1017, se aprobó una nueva Ley de Contrataciones, la cual en su artículo 3.3 estableció en su literal h) que la norma no era de aplicación para las contrataciones cuyos montos sean iguales o inferiores a 3 UIT, vigentes al momento de la transacción. Es decir, se incrementó el supuesto de 
inaplicación de la norma anterior hasta las 3 UIT, lo que permitía una mayor flexibilidad a las contrataciones realizadas por el Estado hasta dicho monto.

El 11 de julio del 2014 se publicó la Ley 30225, cuyo artículo 5, literal a), establece que la normativa de contrataciones del Estado no es aplicable para las contrataciones cuyos montos sean iguales o inferiores a 8 UIT, vigentes al momento de la transacción. Precisa, además, que dichas contrataciones se encuentran sujetas a supervisión por parte del OSCE, supuesto que se materializa con la remisión mensual por parte de las entidades.

En cuanto a la supervisión, la Opinión 128-2017/DTN del OSCE arriba a dos conclusiones. La primera es que las entidades deben registrar y publicar en el Sistema Electrónico de Contrataciones del Estado (SEACE) la información de estas contrataciones realizadas durante el mes, para lo cual el plazo máximo es de hasta diez días hábiles del mes siguiente. La segunda es que las entidades que realicen dichas contrataciones deben efectuarlas de acuerdo con los lineamientos establecidos en sus normas de organización interna, en el marco de los principios que regulan la contratación pública, en razón de lo cual determinarán los mecanismos apropiados para garantizar la eficiencia y transparencia en el uso de los recursos públicos. En consecuencia, las entidades pueden implementar mecanismos similares a los establecidos en la normativa de contrataciones del Estado.

Es en aplicación de este criterio que podemos entender que las entidades públicas emiten las directivas internas para regular las contrataciones de hasta 8 UIT; sin embargo, son pocas las entidades que han llevado a cabo esta tarea, lo que implica que la mayoría de las aproximadamente 2300 entidades públicas desarrollan sus contrataciones de hasta 8 UIT sin tener una base legal clara.

Para elaborar el presente artículo, procedimos a revisar las directivas internas para contrataciones de hasta 8 UIT de las siguientes entidades: OSCE, Ministerio de Economía y Finanzas, EsSalud, Defensoría del Pueblo, Presidencia del Consejo de Ministros, Protransporte, Ministerio de Agricultura, Instituto Nacional de Salud del Niño, Organismo Supervisor de la Inversión en Infraestructura de Transporte de Uso Público (OSITRAN), Gobierno Regional de Arequipa, entre otras. Se pudo advertir que tales directivas incluyen como base legal estas normas: Código de Ética de la Función Pública, Ley de Presupuesto, Código Civil, TUO de la Ley de Contrataciones y el Reglamento de Organización y Funciones de cada entidad. Como se puede apreciar, no figura entre estas el Código Civil, que es una norma diseñada para la contratación entre particulares, y que no es la herramienta adecuada para las contrataciones realizadas por la Administración Pública, al no tener como eje central el interés público y al no considerar el derecho público. Sobre el particular, García de Enterría y Fernández (1986) sostienen:

Por lo pronto, hay que decir que el derecho administrativo es un derecho público, del que constituye una de sus ramas más importantes. Siendo la Administración 
Pública la única personificación interna del Estado, cuyos fines asume, y siendo también dicha persona el instrumento de relación permanente y general con los ciudadanos (en tanto que las funciones no administrativas del Estado son de actuación intermitente y, o bien no se expresan en un sistema de relaciones jurídicas con los ciudadanos, o cuando esto ocurre, tales relaciones afectan solo a contados y excepcionales sujetos), es lícito decir que el derecho administrativo es el derecho público del Estado por excelencia. (pp. 38-39)

Finalmente, es necesario recordar respecto al supuesto de pretender utilizar el Código Civil como base legal para las contrataciones de la Administración Pública lo señalado por Diez (1981), cuando precisa que la Ley Administrativa puede ser de relación o de organización y acción administrativa:

Las leyes administrativas se clasifican teniendo en cuenta su contenido. Hay normas de relación y normas de organización y acción administrativa. Las normas de relación son aquellas que se dictan para delimitar las esferas jurídicas subjetivas de la administración y de los particulares. Son leyes que atribuyen a veces poderes jurídicos y facultades a la Administración Pública, pero también son las que vienen a proteger directamente las situaciones jurídicas de los particulares, convirtiéndolas en derechos subjetivos oponibles frente al Estado. En lo que se refiere a las normas de organización y acción administrativa, son aquellas que determinan, por una parte, la estructura orgánica de la Administración Pública y, por otra, los fines propios que la administración ha de perseguir con su actuación y el modo y forma como esto debe realizarse. Estas normas no se dictan para garantía de los derechos subjetivos de los particulares, sino para la tutela del interés público. (pp. 317-318)

En tal sentido, entendemos que no resulta pertinente pretender utilizar el Código Civil como base legal para las contrataciones públicas de hasta 8 UIT. No obstante, resulta preocupante que en ninguna de las directivas revisadas se pudo encontrar el mecanismo que pueden o deben emplear las partes en caso de que surjan controversias durante la contratación.

\section{EL ARBITRAJE ADMINISTRATIVO}

Desde la publicación de la Ley de Contrataciones del Estado en 1998, el arbitraje ha sido incluido como medio obligatorio de solución de controversias para los conflictos suscitados durante la etapa contractual, supuesto que se ha mantenido hasta la actual Ley de Contrataciones. Por su carácter obligatorio y específico, muchos autores denominan a este medio heterocompositivo de solución de controversias arbitraje administrativo. Kundmüller (s. f.) señala:

En este último caso, el marco normativo administrativo de contrataciones y adquisiciones, en adelante: marco normativo o marco normativo subanálisis, otorga cabida a la conciliación y al arbitraje administrativo en materia de contrataciones 
y adquisiciones, siendo este último medio de solución de controversias al que nos referiremos a partir de ahora, nominándolo simplemente como arbitraje administrativo y analizándolo en el contexto del ámbito de acción de las Administraciones Públicas. (p. 224)

Podemos definir el arbitraje administrativo como el mecanismo mediante el cual la Administración Pública, en cualesquiera de sus manifestaciones, y los administrados pueden pactar que sus diferencias, surgidas en las materias de su libre disposición o en aquellas expresamente señaladas por la ley, sean resueltas por árbitros mediante un laudo que tiene la eficacia de la cosa juzgada, excluyendo así el asunto concreto del conocimiento de los órganos jurisdiccionales competentes.

En esa línea de pensamiento, el OSCE cuenta en su organigrama con la Dirección de Arbitraje Administrativo, que es el órgano encargado de la administración y organización de procesos arbitrales. Entre sus principales funciones, se encuentra participar en calidad de secretaría arbitral (conforme le sea encargada dicha labor por las partes en confrontación o por los árbitros), designar a los árbitros, resolver las recusaciones contra ellos, además de llevar a cabo el proceso de inscripción y renovación en el registro de árbitros del OSCE.

No pretendemos en el presente artículo establecer si el arbitraje administrativo podría constituir una categoría diferente del arbitraje entre privados, o si es una subespecialización del arbitraje; o si se agota en la Ley de Contrataciones o puede encontrarse en otras normas como la Ley de Concesiones o la de Expropiaciones. Solo queremos resaltar que dentro de la normativa de contrataciones se utiliza de manera obligatoria el arbitraje como mecanismo de solución de controversias contractuales, el cual tiene características diferentes del arbitraje regulado por el Decreto Legislativo 1071, Ley de Arbitraje.

En ese sentido, el OSCE, entre las atribuciones que le confiere la Ley de Contrataciones en su artículo 52, tiene la potestad de organizar y administrar arbitrajes, de acuerdo con lo previsto en el reglamento y de conformidad con la directiva que se apruebe para tal efecto. Igualmente, puede designar árbitros y resolver las recusaciones sobre los mismos en arbitrajes que no se encuentren sometidos a una institución arbitral.

Considerando lo señalado, cabe preguntarse si es posible utilizar el arbitraje administrativo como mecanismo de solución de controversias para los conflictos surgidos en las contrataciones de hasta 8 UIT y si corresponde al OSCE dicha designación.

\section{EL CASO MEGAFRANK}

El 10 de abril del 2018, la empresa Megafrank E. I. R. L. solicitó al OSCE la designación de árbitro residual para que resuelva la controversia suscitada con EsSalud respecto a la Orden de Compra 4502539482. En el Oficio 3288-2018-OSCE/DAR, la Dirección de Arbitraje 
del OSCE concluye el procedimiento de designación, argumentando que el importe de la controversia es de 24500 soles, por lo que no resulta de aplicación la Ley de Contrataciones. El 5 de septiembre del 2018, la empresa interpuso un recurso de apelación ante la presidencia del OSCE señalando que, conforme a la Ley de Contrataciones y su reglamento, las controversias en ejecución contractual se resuelven mediante conciliación o arbitraje, no excluyéndose ningún tipo de controversias y menos por razones de cuantía; asimismo, argüía que, de rechazar el pedido de designación de árbitro, resultaba imposible recurrir a otra vía en procura de su derecho de cobro, lo cual le generaba un gran agravio. Mediante la Resolución 101-2018-OSCE-PRE, del 17 de octubre del 2018, se declaró infundada la apelación, tomando como argumento central que, sin importar el objeto de la contratación, si el monto de la misma es inferior a 8 UIT, dicha contratación se encuentra fuera del ámbito de aplicación de la normativa de contrataciones del Estado; adicionalmente, se señala que lo resuelto no causa agravio ni priva de su derecho de cobro, por cuanto tiene expedita la vía judicial o arbitral, conforme al Código Civil o a la Ley de Arbitraje.

Como se puede apreciar, la respuesta del OSCE no resuelve el problema de la empresa Megafrank, que busca un mecanismo especializado y rápido para resolver su controversia. Asimismo, en este caso, considerando que la Orden de Compra 4502539482 no incluye convenio arbitral, solo le queda a la empresa Megafrank acudir a la vía del Poder Judicial para poder cobrar el importe adeudado.

\section{EL ARBITRAJE EN CECONAR}

Al revisar la página web de la Superintendencia Nacional de Salud (SUSALUD), encontramos su Centro de Conciliación y Arbitraje (CECONAR)', que es el organismo autónomo que tiene como misión procurar la solución de las controversias en la prestación de servicios de salud y demás derechos relacionados con este tema, principalmente en el ámbito del aseguramiento universal en salud (AUS) y la seguridad social, a través de mecanismos alternativos como son la conciliación y el arbitraje.

Las principales ventajas que ofrece dicho centro son las siguientes:

- Buen manejo técnico especializado

- Costos accesibles para las partes

- Rapidez de los procesos en comparación con otras instancias

- Neutralidad e imparcialidad en la solución de controversias

- Solución definitiva de las controversias

- Confidencialidad de los procesos

1 Véase la página web: http://portal.susalud.gob.pe/cdi/centro-de-conciliacion-y-arbitraje-ceconar/ 
Como se puede apreciar, estamos ante un centro especializado de solución de controversias respecto a servicios de salud.

Mediante la Resolución de Superintendencia 162-2016-SUSALUD/S, se aprobó el reglamento de CECONAR, que regula las actuaciones arbitrales que se administran en el centro, al que supletoriamente se aplican las normas generales del Decreto Legislativo 1071, que norma el arbitraje. Este reglamento contiene el texto del convenio arbitral tipo de CECONAR, que debe ser promovido y difundido por el centro, así como todo el desarrollo del proceso arbitral.

Como se puede apreciar en el portal de CECONAR, las tarifas arbitrales del centro son las que figuran en la tabla 1.

Tabla 1

Tarifas de los honorarios arbitrales en el CECONAR

\begin{tabular}{clccc}
\hline Modalidad & \multicolumn{1}{c}{ Cuantía } & $\begin{array}{c}\text { Porcentaje } \\
\text { del tope }\end{array}$ & $\begin{array}{c}\text { Honorarios } \\
\text { total }\end{array}$ & $\begin{array}{c}\text { Honorario } \\
\text { por parte }\end{array}$ \\
\hline A & Hasta $\$ 500$ & 20 & $\$ 100$ & $\$ 50$ \\
B & Sobre $\$ 500$ y hasta $\$ 1000$ & 15 & $\$ 150$ & $\$ 75$ \\
C & Sobre $\$ 1000$ y hasta $\$ 1500$ & 12 & $\$ 180$ & $\$ 90$ \\
D & Sobre $\$ 1500$ y hasta $\$ 2000$ & 11,50 & $\$ 230$ & $\$ 115$ \\
E & Sobre $\$ 2000$ y hasta $\$ 3000$ & 10 & $\$ 300$ & $\$ 150$ \\
F & Sobre $\$ 3000$ y hasta $\$ 4000$ & 9 & $\$ 360$ & $\$ 180$ \\
G & Sobre $\$ 4000$ y hasta $\$ 5000$ & 8 & $\$ 400$ & $\$ 200$ \\
H & Sobre $\$ 5000$ y hasta $\$ 6000$ & 7,50 & $\$ 450$ & $\$ 225$ \\
I & Sobre $\$ 6000$ y hasta $\$ 7000$ & 7,14 & $\$ 500$ & $\$ 250$ \\
J & Sobre $\$ 7000$ y hasta $\$ 10000$ & 6 & $\$ 600$ & $\$ 300$ \\
K & Sobre $\$ 10000$ y hasta $\$ 20000$ & 5 & $\$ 1000$ & $\$ 500$ \\
L & Sobre $\$ 20000$ y hasta $\$ 50000$ & 3 & $\$ 1500$ & $\$ 750$ \\
M & Sobre $\$ 50000$ y hasta $\$ 100000$ & 2 & $\$ 2000$ & $\$ 1000$ \\
N & Sobre $\$ 100000$ y hasta $\$ 500000$ & 0,50 & $\$ 2500$ & $\$ 1250$ \\
O & Sobre $\$ 500000$ y hasta $\$ 1000000$ & 0,30 & $\$ 3000$ & $\$ 1500$ \\
O & Sobre $\$ 1000$ 000 & & $\$ 3600$ & $\$ 1800$ \\
\hline
\end{tabular}

Fuente: Reglamento y tabla de Aranceles y Honorarios aprobado por Resolución de Superintendencia 050-99-SEPS (2017, p. 3)

Como se observa, para controversias de hasta 8 UIT, la tarifa es de 600 dólares, la cual debe ser pagada $50 \%$ por cada parte. 


\section{ALTENATIVA PROPUESTA}

El artículo 52 de la Ley de Contrataciones establece, entre otras, las siguientes funciones del OSCE:

a) Velar y promover que las entidades realicen contrataciones eficientes, bajo los parámetros de la ley, su reglamento y normas complementarias, la maximización del valor de los fondos públicos y la gestión por resultados. [...]

f) Emitir directivas, documentos estandarizados y documentos de orientación en materia de su competencia. [...]

k) Organizar y administrar arbitrajes de acuerdo a lo previsto en el reglamento y de conformidad con la directiva que se apruebe para tal efecto.

l) Designar árbitros y resolver las recusaciones sobre los mismos en arbitrajes que no se encuentren sometidos a una institución arbitral.

El Reglamento de Organización y Funciones del OSCE (ROF), en su artículo 99, establece que la Dirección de Arbitraje tiene como finalidad desarrollar las actividades relacionadas con los medios de solución de controversias durante la etapa de ejecución contractual en el marco de las contrataciones del Estado; y en el artículo 101 le asigna las siguientes funciones, entre otras:

n) Proponer los proyectos de normas especializadas en materia de solución de controversias en la etapa de ejecución contractual en el marco de la normativa de contrataciones del Estado. [...]

q) Proponer las directivas de gestión en materia de su competencia, así como velar por su cumplimiento para la optimización de los procesos del OSCE.

El artículo 103 del ROF plantea las siguientes funciones de la Subdirección de Procesos Arbitrales: organizar y administrar los arbitrajes a cargo del OSCE, conforme a lo establecido en el Reglamento del Sistema Nacional de Arbitraje - OSCE, y en aquellos que corresponda, con el apoyo de las otras subdirecciones de ser el caso.

Debemos considerar que, si bien el artículo 5 de la Ley de Contrataciones establece que las contrataciones de hasta 8 UIT están excluidas del ámbito de aplicación de la misma, tal supuesto no exime a las entidades de estar bajo la supervisión del OSCE, lo que se evidencia al dar cumplimiento a la Directiva 008-2017-OSCE/CD, "Disposiciones aplicables al registro de información en el Sistema Electrónico de Contrataciones del Estado - SEACE".

En tal sentido, no encontramos impedimento legal alguno para que el OSCE, a través de la Dirección de Arbitraje y de acuerdo con las funciones antes descritas, elabore un procedimiento arbitral especial para resolver las controversias suscitadas en las contrataciones de hasta 8 UIT, emulando el esquema de DIRECOMAR, para lo cual inclusive podría valerse del Sistema Nacional de Arbitraje (SNA) del OSCE. Este procedimiento puede ser aprobado mediante una resolución de la presidencia del OSCE y ser regulado 
a través de una directiva, por lo que no sería necesario un cambio ni en la ley ni en el reglamento.

Los elementos que debe comprender la directiva propuesta son los siguientes:

a. Disposiciones generales

b. Ámbito de aplicación

c. Funciones del SNA

d. Cláusula arbitral tipo que deberá incluirse en las órdenes de compra o en las órdenes de servicio

e. Régimen arbitral

f. Sede arbitral, la cual será el local del OSCE

g. Designación arbitral, a través de árbitro único del Registro Nacional de Árbitros

h. Tasa única arbitral (sugerimos la mitad de 1 UIT) que será cancelada por el demandante y, de ser el caso, reconocida en el laudo arbitral

i. El SNA del OSCE será la secretaría arbitral

j. Actuaciones arbitrales

k. Duración máxima del proceso arbitral desde la instalación: treinta días hábiles, incluida la audiencia única y emisión de laudo

\section{CONCLUSIONES}

Podemos concluir que, si bien la Ley de Contrataciones debe ser utilizada por todas las entidades y empresas del Estado, contempla también supuestos excluidos de su aplicación, entre los cuales encontramos a las contrataciones de hasta 8 UIT. Estas contrataciones, solo en algunos casos, están reguladas a través de directivas internas de cada entidad, pero la mayoría de veces no tienen regulación, lo cual implica que muchas entidades de manera errónea están utilizando el Código Civil para regular contrataciones que incluyen interés público. Lo más preocupante es que tampoco existe un procedimiento adecuado para resolver las controversias suscitadas en la etapa contractual de las contrataciones de hasta 8 UIT, lo que significa no solo el desconcierto en las partes contratantes sobre qué medio de solución de controversias utilizar, sino que también está generando una sensación cada vez más grande de indefensión en los contratantes.

En ese sentido, y a efectos de resolver el problema descrito, se plantea, dentro de los alcances de la normativa de contrataciones y la normativa interna del OSCE, un arbitraje especial que se encargue de las controversias de las contrataciones de hasta 8 UIT, el 
cual será obligatorio, expedito y económico para las partes, y que utilizará la organización con la que ya cuenta el OSCE para tal fin, lo que implica que no erogará mayores recursos económicos del Estado.

\section{REFERENCIAS}

Amado, J., y Miranda, L. (2000). Aplicabilidad de la cláusula pacta sunt servanda del artículo 62 de la Constitución a los contratos regulados por el derecho público. Thémis, 40, 255-262.

De la Puente, M. (1991). El contrato en general (t. 1). Pontificia Universidad Católica del Perú, Fondo Editorial.

Diez, M. M. (1981). Manual de derecho administrativo (t. 1). Plus Ultra.

García de Enterría, E., y Fernández, T. R. (1986). Curso de derecho administrativo (t. 1). Civitas.

Kundmüller, F. (s. f.). El arbitraje administrativo en contrataciones y adquisiciones del Estado. Revista Peruana de Derecho de la Empresa, 221-246. http://blog.pucp. edu.pe/blog/stein/wp-content/uploads/sites/734/2019/03/El-ArbitrajeAdministrativo-Kundmuller-Per\%C3\%BA.pdf

Linares, M. (2006). El contrato administrativo en el Perú. Revista de Derecho Administrativo, $1,285-308$.

Reglamento y Tabla de Aranceles y Honorarios aprobado por Resolución de Superintendencia 050-99-SEPS. 2 de enero del 2017. http://portal.susalud. gob.pe/wp-content/uploads/archivo/ceconar/Aranceles-administrativos-yhonorarios-arbitrales.pdf

Resolución 101-2018-OSCE-PRE [Organismo Supervisor de las Contrataciones del Estado]. 17 de octubre del 2018. https://cdn.www.gob.pe/uploads/document/ file/1128587/RESOLUCION_N\%C2\%BA_101-2018-OSCE-PRE20200731-10789418r309i.pdf

Salazar, R. (2009). Las formas jurídicas administrativas y la contratación pública sobre bienes, servicios y obras. Revista de Derecho Administrativo, 7, 24-35.

Trelles, 0. (2002). El contrato administrativo, el contrato-ley y los contratos de concesión de servicios públicos. Thémis, 44, 237-251. 